\title{
Concurrent design of an active vibration feedback controller and actuator/sensor selection for a composite plate
}

\author{
Taranjitsingh Singh*, Wilm Decré*, Jan Swevers*, Goele Pipeleers*
}

\begin{abstract}
This article presents two approaches to simultaneously select optimal locations of sensors and actuators and design of a $\mathcal{H}_{\infty}$ dynamic output feedback controller. The first approach discussed in this paper is based on an iterative reweighted $\ell_{1}$ norm regularization, an heuristic approach. The second approach converts the combined design into mixed boolean semi-definite programming optimization problem (MBSDP). A branch and bound (BNB) algorithm is adopted to solve this convex optimization problem to give a global optimal solution in terms of selection and control design. The two approaches are compared with an exhaustive search in terms of optimality and is applied to a vibration rejection control design of a composite plate.
\end{abstract}

\section{INTRODUCTION}

As the computation power of the digital control systems keeps increasing, more industries have started adopting complex $\mathcal{H}_{\infty}$ feedback control design for linear time-invariant (LTI) systems. However, in multi input multi output (MIMO) systems, if the number of inputs and outputs increases, the complexity of the controller also increases. Apart from keeping up with the complex behaviour, using less hardware resources, i.e. using less sensors and actuators would be economically beneficial for industries. Hence, it would be advantageous to design controllers with acceptable closedloop performance with minimum hardware resources.

In order to find the perfect combination of sensors and actuators from all the possible candidates, an exhaustive search is required to get the global solution. However, doing an exhaustive search could become computationally expensive in case of large numbers of possible sensors and actuators. Suppose there are $n_{s}$ possible sensors and $n_{a}$ possible actuator locations, it would require an exhaustive search for $\left(2^{n_{a}}-1\right)\left(2^{n_{a}}-1\right)+1$ combinations. So, the number of possible combinations increases exponentially with the number of sensors and actuators. We need a computationally less expensive approach to find the best combination of hardware along with acceptable closed-loop performance.

Initially, controller-independent strategies such as selection of sensors and actuators based on open loop $\mathcal{H}_{2} / \mathcal{H}_{2}$ norms and evaluating observability and controllability conditions were considered [1], [2]. However, selection of sensors and actuators based on open loop systems may not provide the optimal selection if the focus is on the closed loop performance.

*T. Singh, W.Decré, J. Swevers and G. Pipeleers are with the Department of Mechanical Engineering, Div. PMA, KU Leuven, and also affiliated with DMMS lab, Flanders Make, Celestijnenlaan 300B, B-3001, Heverlee, Belgium. Email: firstname.lastname@kuleuven.be
Many controller-dependent selection strategies are discussed and elaborated in [1]. Researches [3] and [4] use heuristics techniques like genetic algorithm in combination with linear quadratic regulator (LQR) and applied on active suspension control of vehicles. However, ricatti based LQR techniques provides optimal controller, linear matrix inequalities (LMI) based $\mathcal{H}_{\infty}$ optimal design have the ability to account for model uncertainty and perform a robust control design. Combined $\mathcal{H}_{\infty}$ feedback controller with optimal selection is formulated as a mixed boolean semi-definite programming (MBSDP) problem in [5] and [6], where the mixed termed are separated to form an optimization problem. These methods do not attain global optimality in selection and closed loop performance simultaneously.

In this article, two new approaches are discussed to simultaneously design $\mathcal{H}_{\infty}$ controller with optimal sensor and actuator selection. The first method, is to formulate the combinatorial problem and impose sparsity with $\ell_{0}$-quasi norms. The convex approximations in terms of (weighted) $\ell_{1}$ norms are preferred over the non-convex $\ell_{0}$ terms [7], [8]. This technique is used for state feedback and static output feedback control design [9] and [10]. This approach is extended to dynamic output feedback in [11]. A similar approach is presented in [12] but that approach is based on complex LMIs and more conservative controller synthesis.

The second approach discussed in this paper reformulates the concurrent design problem as a MBSDP problem, where the discrete decision variables determine which particular sensor and actuator is selected and $\mathcal{H}_{\infty}$ controller synthesis are represented as SDP variables. Big-M reformulations results into decoupling the mixed variables to form a convex optimization problem [13] which can be solved using a branch and bound (BNB) algorithm. This approach is introduced in [14], but do not discuss any potential application of the approach. An comparison of the two methods with an exhaustive search are done in [15], however, they design a $\mathcal{H}_{\infty}$ static output feedback controller focused on closed loop stability of unstable distributed networks and they do not consider closed loop performances.

The two approaches discussed in our paper are applied to a vibration rejection control of a smart composite plate along with the optimal actuator selection. They are compared with an exhaustive search for the same application in order to analyze the optimality of the solution and computation time required to attain the solution. The vibration control application is discussed in [16], however it is for a linear parameter varying (LPV) system and the number of sensors and actuators is not very high. 
We have organized the paper in the following way. Section II briefly describes $\mathcal{H}_{\infty}$ controller synthesis and describes the control design problem as an optimization problem. Section III combines the LMI conditions for the simultaneous control design. Here, the two approaches are briefly discussed: the iterative reweighted $\ell_{1}$-norm algorithm and the MBSDP with BNB algorithm. These two approaches are applied to design a feedback controller combined with optimal actuator selection for the active vibration control of a smart composite plate in the section IV. And finally, section $\mathrm{V}$ concludes the article.

\section{II. $\mathcal{H}_{\infty}$ FEEDBACK CONTROL OF A LTI SYSTEM}

Consider a strictly proper, continuous-time LTI plant $\mathcal{P}$

$$
\mathcal{P}:\left\{\begin{array}{l}
\dot{x}(t)=A x(t)+B_{w} w(t)+B_{u} u(t) \\
z(t)=C_{z} x(t)+D_{z w} w(t)+D_{z u} u(t) \\
y(t)=C_{y} x(t)+D_{y w} w(t)+D_{y u} u(t)
\end{array}\right.
$$

Here, $x \in \mathbb{R}^{n_{x}}, w \in \mathbb{R}^{n_{w}}, u \in \mathbb{R}^{n_{u}}, z \in \mathbb{R}^{n_{z}}, y \in$ $\mathbb{R}^{n_{y}}$ represents the plant state vector, the exogneous input, control input, the regulated output and the measured output respectively.

$$
\mathcal{K}:\left\{\begin{array}{l}
\dot{\zeta}(t)=A_{C} \zeta(t)+B_{C} y(t) \\
u(t)=C_{C} \zeta(t),
\end{array}\right.
$$

for obtaining a LTI controller $\mathcal{K}$ such that $\zeta(t)$ represents the controller state vector.

If $\mathcal{T}$ is the closed-loop transfer function from $w$ to $z$, then the closed-loop specifications are formulated using the $\mathcal{H}_{\infty}$ norm of $\mathcal{T}$ which is shown by

$$
\|\mathcal{T}\|_{\infty}=\sup _{\|w\|_{2} \neq 0} \frac{\|z\|_{2}}{\|w\|_{2}}
$$

such that $\|w\|_{2}=\left[\int_{0}^{\infty} w^{T}(t) w(t) d t\right]^{1 / 2}$ is a 2-norm of a continuous signal. The combined design approaches are based on these LMI formulations for $\mathcal{H}_{\infty}$ controller synthesis which is detailed below [14] (Refer [17] for proof).

Lemma 2.1: There exists a dynamic output feedback controller (2) that yields $\|\mathcal{T}\|_{\infty}<\gamma$ if, and only if there exists symmetric matrices $X, Y \in \mathbb{R}^{n_{x} \times n_{x}}$ for which,

$$
\left[\begin{array}{cccc}
H e\left(A X+B_{u} \hat{C}_{C}\right) & \star & \star & \star \\
\hat{A}_{C}+A^{T} & H e\left(Y A+\hat{B}_{C} C_{y}\right) & \star & \star \\
B_{w}^{T} & Y B_{w}+\hat{B}_{C} D_{y w} & -I & \star \\
C_{z} X+D_{z u} \hat{C}_{C} & C_{z} & D_{z w} & -\Gamma I
\end{array}\right] \prec
$$

and

$$
\left[\begin{array}{cc}
X & I \\
I & Y
\end{array}\right] \succ 0
$$

where $H e(X)=X+X^{T}$ for a symmetric matrix $X$, $X \succ 0(X \prec 0)$ denotes the positive-definiteness (negativedefiniteness) of $X$, a $\star$ indicate terms to make a LMI formulation symmetric, $I$ represents identity matrix of a suitable dimension inferred from the context and $\Gamma=\gamma^{2}$. $\hat{A}_{C} \in \mathbb{R}^{n_{x} \times n_{x}}, \hat{B}_{C} \in \mathbb{R}^{n_{x} \times n_{y}}$ and $\hat{C}_{C} \in \mathbb{R}^{n_{u} \times n_{x}}$ are slack variables procured using linearizing change of variables (see [17] for more details). The control design optimization problem is defined as

$$
\begin{array}{cl}
\underset{X, Y, \hat{A}_{C}, \hat{B}_{C}, \hat{C}_{C}, \Gamma}{\operatorname{minimize}} & \Gamma \\
\text { subject to: } & (4),(5) .
\end{array}
$$

The controller (2) is constructed using (7).

$$
\begin{aligned}
C_{C}= & \hat{C}_{C} M^{-T} \\
B_{C}= & N^{-1} \hat{B}_{C} \\
A_{C}= & N^{-1}\left(\hat{A}_{C}-N B_{C} C_{y} X\right. \\
& \left.-Y B_{u} C_{C} M^{T}-Y A X\right) M^{-T} .
\end{aligned}
$$

where $M, N \in \mathbb{R}^{n_{x} \times n_{x}}$ are full-rank nonsingular matrices which satisfies

$$
M N^{T}=I-X Y
$$

\section{COMBINED DESIGN PROBLEM}

On observing (7), it can be inferred that the number on non-zero rows of $C_{C}$ indicates which actuators are used in a control design and similarly the number of non-zero columns of $B_{C}$ decides which sensors to be fed back for the control design. Mathematically, $\left\|\hat{C}_{C}\right\|_{r-\ell_{0}}$ (and hence $\left\|C_{C}\right\|_{r-\ell_{0}}$ ) and $\left\|\hat{B}_{C}\right\|_{c-\ell_{0}}$ (and hence $\left\|B_{C}\right\|_{c-\ell_{0}}$ ), which are the $\ell_{0}$-quasi norm, represents the number of non-zero rows and columns of $C_{C}$ and $B_{C}$ respectively. Finding the nonzeros rows and columns of the controller matrices gives rise to MBSDP problem, where the selection variables are the boolean variables and is solved using two different strategies.

\section{A. Iterative reweighted $\ell_{1}$-norm algorithm}

In order to yield a convex optimization problem to be solved in a polynomial time, the $\ell_{0}$-quasi norms, being hard combinatorial problem are relaxed by $\ell_{1}$ norms to formulate a convex optimization problem [8]. A more powerful relaxation is a weighted $\ell_{1}$-quasi norms of the controller matrices [18]. These assumptions give rise to an iterative multi-objective optimization problem to form the combined MBSDP design problem which is given by (9).

$$
\begin{array}{ll}
\underset{x, Y, \hat{A}_{C}, \hat{B}_{C}, \hat{C}_{C}, \Gamma}{\operatorname{minimize}} & \Gamma+\eta_{b}\left\|\hat{B}_{C} W_{b}^{k}\right\|_{c-\ell_{1}}+\eta_{c}\left\|W_{c}^{k} \hat{C}_{C}\right\|_{r-\ell_{1}} \\
\text { subject to: } & (4),(5) .
\end{array}
$$

$\prec 0$ Here, $\eta_{b}, \eta_{c}>0$ are the tuning parameters regulating the column sparsity of $\hat{B}_{C}$ and row sparsity of $\hat{C}_{C}$ respectively. $W_{b}^{k}=\operatorname{diag}\left[W_{b, i}^{k}\right]$ and $W_{c}^{k}=\operatorname{diag}\left[W_{c, j}^{k}\right]$ are the weights updated at every $k$ iterations for $i^{\text {th }}$ column and $j^{\text {th }}$ row and are shown in (10),

$$
W_{b, i}^{k}=\frac{1}{\left\|\hat{B}_{C, i}^{k-1}\right\|_{\ell_{1}}+\epsilon}, \quad W_{c, j}^{k}=\frac{1}{\left\|\hat{C}_{C, j}^{k-1}\right\|_{\ell_{1}}+\epsilon}
$$

such that $\left\|\hat{B}_{C, i}^{k-1}\right\|_{\ell_{1}}$ represents the $\ell_{1}$ norm of the $i^{\text {th }}$ column and $\left\|\hat{C}_{C, j}^{k-1}\right\|_{\ell_{1}}$ denotes the $\ell_{1}$ norm of the $j^{t h}$ row. $\epsilon$ is a tuning parameter where $0<\epsilon \ll 1$ in order to avoid infinite $W_{b, i}^{k}$ and $W_{c, j}^{k}$ in iterations. The optimal solution is obtained by adopting the Algorithm 1. [11]. 


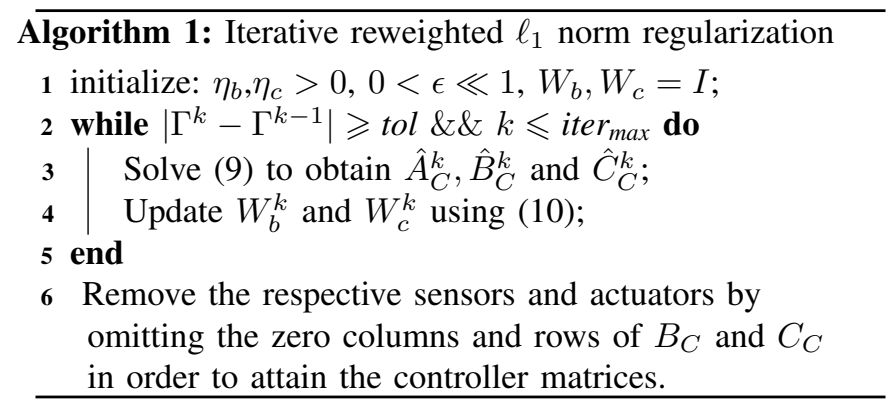

\section{B. MBSDP with BNB}

Let $\hat{B}_{C} \Phi$ and $\Psi \hat{C}_{C}$ be the mixed boolean semi-definite terms that expresses sensor and actuator selection respectively, where $\Phi$ and $\Psi$ are the diagonal matrices consisting of boolean variables given as

$$
\Phi=\left[\begin{array}{ccc}
s_{1} & & 0 \\
& \ddots & \\
0 & & s_{n_{y}}
\end{array}\right], \Psi=\left[\begin{array}{ccc}
s_{1} & & 0 \\
& \ddots & \\
0 & & s_{n_{u}}
\end{array}\right] .
$$

Here, $s_{i}$ and $a_{j}$ are the decision variables for $i^{t h}$ sensor and $j^{\text {th }}$ actuator respectively such that we obtain the set $\Delta=$ $\left\{s, a \mid s_{i}, a_{j} \in\{0,1\}\right\}$ determining all possible candidates of sensors and actuators. When these boolean diagonal matrices are introduced in (4), mixed boolean semi-definite terms occur due to the non-linear coupling, thus resulting into a non-convex optimization problem. The mixed variables can be decoupled by applying Big-M reformulations, such that the boolean constraints are transformed into the new constraints [13] and are given by

$$
m_{B} \Phi \leqslant \hat{B}_{C} \leqslant M_{B} \Phi, \quad \Psi m_{C} \leqslant \hat{C}_{C} \leqslant \Psi M_{C} .
$$

Here, $m_{B} \in \mathbb{R}_{-}^{n_{x} \times n_{y}}, M_{B} \in \mathbb{R}_{+}^{n_{x} \times n_{y}}, m_{C} \in \mathbb{R}_{-}^{n_{u} \times n_{x}}$ and $M_{C} \in \mathbb{R}_{+}^{n_{u} \times n_{x}}$ represents the Big-M bound matrices for $\hat{B}_{C}$ and $\hat{C}_{C}$. The Big-M reformulation is identical to the original constraint, if $M_{B}$ and $M_{C}$ are selected adequately larger and $m_{B}$ and $m_{C}$ are selected adequately smaller than any possible value of $\hat{B}$ and $\hat{C}$ respectively.

If there are additional hardware requirements, for an instance, $\left\{k \in\left[1, n_{y}\right]\right\}$ sensors and $\left\{l \in\left[1, n_{u}\right]\right\}$ actuators, can be easily considered as

$$
\operatorname{Tr}(\Phi)=k, \quad \operatorname{Tr}(\Psi)=l,
$$

where $\operatorname{Tr}(X)$ determines the trace of a symmetric matrix $X$.

In order to find an optimal set $\Delta^{\star}=\left\{s^{\star}, a^{\star} \mid \Delta^{\star} \subseteq\right.$ $\Delta\}$ satisfying both closed loop specifications and hardware constraints for the control design, the following MBSDP optimization is considered

$$
\begin{array}{cl}
\underset{X, Y, \hat{A}_{C}, \hat{B}_{C}, \hat{C}_{C}, \Gamma, s^{\star}, a^{\star}}{\operatorname{minimize}} & \Gamma \\
\text { subject to: } & (4),(5),(12),(13),
\end{array}
$$

which results into a dynamic output feedback controller of the form (2) for (1), with $\|\mathcal{T}\|_{\infty}<\gamma$ and optimal, $\Delta^{\star}$ for desired $k$ sensors and $l$ actuators.

\section{CAse Study}

For validation and comparison of the strategies discussed in the previous section, a composite plate is considered with free-free suspension.

\section{A. Modeling description of a plate.}

The lay-up of $\left[-45^{\circ} 45^{\circ} 0^{\circ} 90^{\circ}\right]_{s}$ is considered for a plate with unidirectional carbon fiber laminates [19]. The plate's size is $500 \mathrm{~mm} \times 400 \mathrm{~mm} \times 2.5 \mathrm{~mm}$. The plate is integrated with 25 M2814-P1 macro-fiber composite (MFC) transducers (actuators) of size $28 \mathrm{~mm} \times 14 \mathrm{~mm} \times 0.3 \mathrm{~mm}$ along with 25 accelerometers (sensors) and their placements is determined by Fig. 1. The dynamics of the accelerometers is ignored in modeling because of their weight being negligible (5 gms per unit) compared to the weights of the actuators.

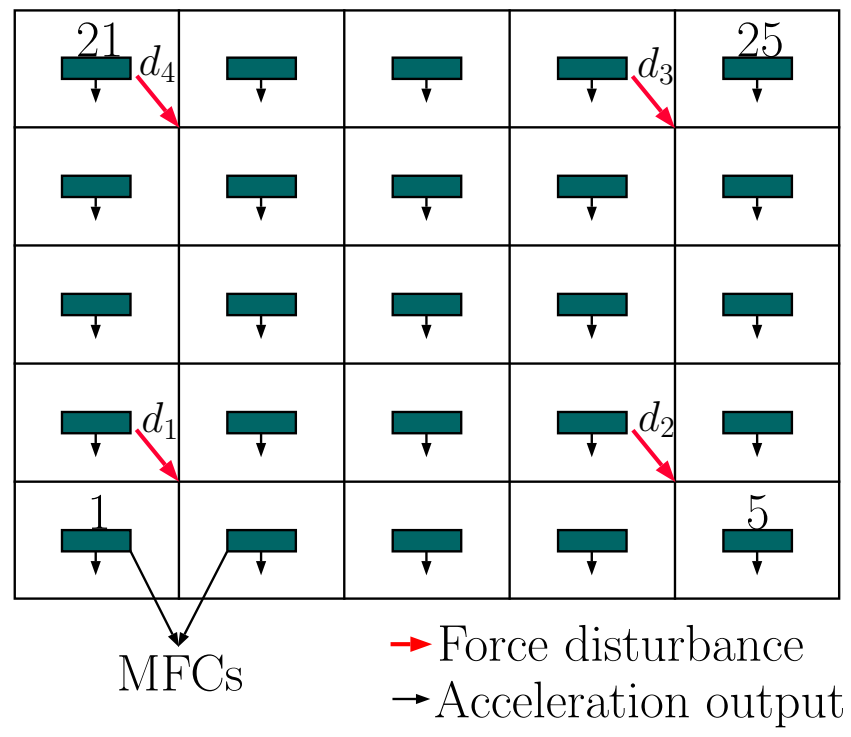

Fig. 1: Simplified model of a composite plate $\left(d_{1}-d_{4}\right.$ represents the force disturbances.)

TABLE I: Parameters used in modeling of the composite plate integrated with MFC actuators.

\begin{tabular}{|c||c||c|}
\hline Parameter & MFC $-\mathbf{d 3 3}$ & laminates \\
\hline mass density & $5440 \mathrm{Kg} / \mathrm{m}^{3}$ & $1500 \mathrm{Kg} / \mathrm{m}^{3}$ \\
$Y_{11}$ & $27.142 \mathrm{GPa}$ & $110.15 \mathrm{GPa}$ \\
$Y_{22}$ & $14.8 \mathrm{GPa}$ & $7.868 \mathrm{GPa}$ \\
$G_{12}$ & $4.1312 \mathrm{GPa}$ & $4.05 \mathrm{GPa}$ \\
$\nu_{12}$ & 0.2922 & 0.26 \\
$G_{13}$ & $5.385 \mathrm{GPa}$ & - \\
$G_{23}$ & $10.5 \mathrm{GPa}$ & - \\
$d_{33}$ & $272 \mathrm{pm} / \mathrm{V}$ & - \\
$d_{32}$ & $-133 \mathrm{pm} / \mathrm{V}$ & - \\
$C_{p}$ & $0.30 \mathrm{nF} / \mathrm{cm}^{2}$ & - \\
\hline
\end{tabular}

The modeling is achieved using equivalent substrate modeling (ESM) [19], and the parameters used in modeling are 
shown in Table I, where $Y_{i j}, G_{i j}, \nu_{i j}$ and $d_{i j}$ represents the Young's moduli, shear moduli, Poisson's ratio and piezoelectric charge constants in $i j$ plane leading to the following dynamic model (Refer [19] for details on ESM).

$$
M_{p} \ddot{q}+C_{d} \dot{q}+\underbrace{\left(K-\Theta C_{p}^{-1} \Theta^{T}\right)}_{K^{g}} q=\Theta u+L d
$$

where $q$ stands for the displacement, $M_{p}, C_{d}$ and $K$ represents the mass, damping and spring matrices respectively. $K^{g}$ is the augmented stiffness matrix generated by piezoelectric stiffness and $C_{p}$ and $\Theta$ represents the capacitance of the MFC actuator and piezoelectric coupling matrix respectively with input voltage $u$ and $d$ as the force disturbances and $L$ represents the localization matrix of the disturbances. Second-Order-Rational-Arnoldi approach [20] reduces the model (15) to 5 vibrational modes to give the following reduced model

$$
M_{p_{r}} \ddot{q}+C_{d_{r}} \dot{q}+K_{r}^{g} q=\Theta_{r} u+L_{r} d,
$$

where the matrices with subscript $(.)_{r}$ represents the respective matrices of the reduced-order model. The corresponding $10^{t h}$ order state space model is given by:

$$
\begin{aligned}
{\left[\begin{array}{c}
\dot{q} \\
\ddot{q}
\end{array}\right]=} & \underbrace{\left[\begin{array}{cc}
0 & I \\
-M_{p_{r}}^{-1} K_{r}^{g} & -M_{p_{r}}^{-1} C_{r}
\end{array}\right]}_{A}\left[\begin{array}{c}
q \\
\dot{q}
\end{array}\right]+\underbrace{\left[\begin{array}{c}
0 \\
-M_{p_{r}}^{-1} \Theta_{r}
\end{array}\right]}_{B_{u}} u \\
& +\underbrace{\left[\begin{array}{c}
0 \\
-M_{p_{r}}^{-1} L_{r}
\end{array}\right]}_{B_{d}} d .
\end{aligned}
$$

Acceleration output $y$ is taken as,

$$
y=\left[\begin{array}{ll}
0 & C_{a}
\end{array}\right]\left[\begin{array}{c}
\dot{q} \\
\ddot{q}
\end{array}\right]
$$

such that substituting (17) into (18) yields

$$
y=\underbrace{\left[\begin{array}{cc}
0 & C_{a}
\end{array}\right] A}_{C}\left[\begin{array}{c}
q \\
\dot{q}
\end{array}\right]+\underbrace{\left[\begin{array}{ll}
0 & C_{a}
\end{array}\right]\left[\begin{array}{ll}
B_{u} & B_{d}
\end{array}\right]}_{D}\left[\begin{array}{l}
u \\
d
\end{array}\right] .
$$

\section{B. $\mathcal{H}_{\infty}$ feedback control design}

Figure. 2 shows the control configuration for a disturbance rejection control design. $W_{D}$ and $W_{U}$ are the loop-shaping filters for the disturbance channel $D$ and the input sensitivity channel $U$, respectively. The goal is to minimize cost function representing the closed-loop specification,

$$
\text { minimize: }\left\|\left[\begin{array}{l}
W_{D} D \\
W_{U} U
\end{array}\right]\right\|_{\infty} \text {. }
$$

The weights $W_{D}$ and $W_{U}$ are chosen as static gains and are shown as

$$
W_{D}=10 I_{25}, \quad W_{U}=35 I_{25}
$$

for each performances $z_{1} \ldots z_{25}$ and $z_{u_{1}} \ldots z_{u_{25}}$ respectively.

Using the system model (16) with the weights (21) and formulating the control configuration as shown in the figure. 2 we attain the plant of the form (1) leading to the optimization problem (6) which is formulated as a control design problem

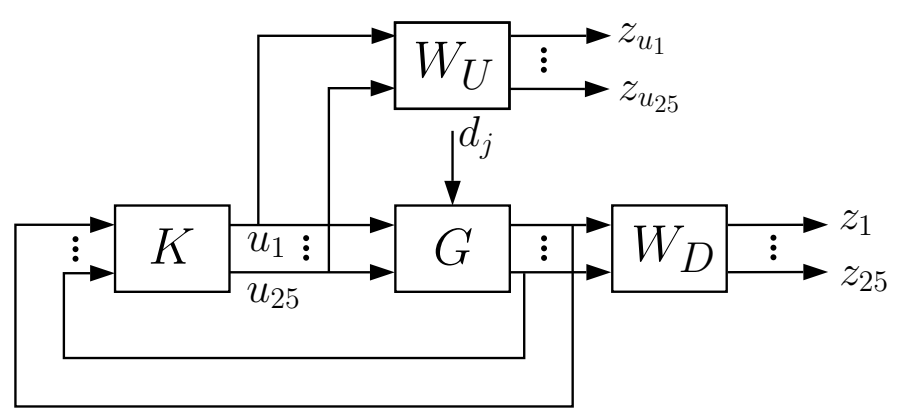

Fig. 2: control configuration for disturbance rejection control design problem with $d_{1} \ldots d_{4}$ disturbances and 25 sensors and actuators.

using an open source toolbox with in-built routines for MATLAB called 'LCToolbox' ${ }^{1}$ [21]. This returns a $\mathcal{H}_{\infty}$ dynamic feedback controller of the form (2), where the LMIs are parsed using YALMIP [22] and MOSEK [23] is used to solve the SDP optimization problem. The upper bound on the closed-loop performance, achieved as a solution from the optimization problem is $\gamma=74.5045$. The resulting controller is a $10^{t h}$ full-order controller which uses all the 25 sensors and actuators.

\section{Concurrent control design with optimal actuator selection}

For our first goal, we focus on obtaining a feedback controller combined with optimal actuator selection. In order to do that, two strategies discussed in the previous section are considered. Both of these strategies are compared in terms of optimality and the computation time.

Firstly, the optimization problem (9) is considered with $\eta_{b}=0$. Different values of $\eta_{c}$ would lead to distinct optimal selections of actuators along with control design. However, for comparison between the strategies, a particular value of the tuning parameter $\eta_{b}=0.75$ is considered. With the values, $\epsilon=10^{-5}$, tol $=10^{-4}$, and maximum number of iterations to be 250 , the algorithm. 1 is solved. This results in selecting 6 actuators at the locations $\{1,2,3,6,11,25\}$ with the upper bound on closed-loop performance as $\gamma=$ 165.4361.

Finally, in order to proceed with the second approach, the matrices that represents the Big-M reformulations i.e, $m_{C}$ and $M_{C}$ are selected as

$$
m_{C}=10 \min \left(\hat{C}_{C}(:)\right), \quad M_{C}=10 \max \left(\hat{C}_{C}(:)\right)
$$

where $\hat{C}_{C}(:)$ represents all the elements in vector of the matrix $\hat{C}_{C}$, with a safety factor of 10 of the matrix's maximum and minimum values.

Here, we assume that 6 numbers of actuators are desired while feeding back all the sensors for the control design. We impose an extra constraint of fixing the actuator at the $1^{\text {st }}$ location as can be seen in figure. 1 . Thus we attain the following optimization problem

${ }^{1}$ https://github.com/meco-group/lc_toolbox 


$$
\begin{array}{cl}
\underset{X, Y, \hat{A}_{C}, \hat{B}_{C}, \hat{C}_{C}, \Gamma, a^{\star}}{\operatorname{minimize}} & \Gamma \\
\text { subject to: } & (4),(5) \\
& \Psi m_{c} \leqslant \hat{C}_{C} \leqslant \Psi M_{C} \\
& \left\{a_{1}\right\}=1, \\
& \operatorname{Tr}(\Psi)=6 .
\end{array}
$$

This gives rise to ${ }^{24} C_{5}$ combinations of actuators distributed among the plate. The optimization problem (23) is parsed using YALMIP and solved with MOSEK, with using YALMIP's inbuilt BNB algorithm. The optimal actuator locations achieved are 1,2,6,7,22 and 25 (see figure. 1). The upper bound on the closed-loop performance is achieved as $\gamma=112.8167$.

In order to compare the optimality and the computation time, an exhaustive search for all the possible combinations is considered. The comparison of both the approaches are shown in the table. II. All the simulations are executed in MATLAB 2015b on a 4-core (8 logical processors) with 2.70GHz Intel Core i7-6820 CPU with 16GB RAM. For performing an exhaustive search, which involves solving all the ${ }^{24} C_{5}$ combinations, the control design problems were distributed parallel among 6 logical processors, while the methods mentioned in this paper uses only one logical processor.

TABLE II: Comparison of the two approaches with an exhaustive search.

\begin{tabular}{|c|c|c|c|}
\hline Approach & $\gamma$ & $\begin{array}{c}\text { Global } \\
\text { optimal }\end{array}$ & $\begin{array}{c}\text { Computation } \\
\text { time (h) }\end{array}$ \\
\hline $\begin{array}{c}\text { Iterative } \\
\text { reweighted } \\
\ell_{1} \text { algortihm }\end{array}$ & 165.4361 & $\times$ & 1.4416 \\
\hline $\begin{array}{c}\text { MBSDP } \\
\text { with BNB } \\
\text { algorithm }\end{array}$ & 112.8167 & $\checkmark$ & 1.5824 \\
\hline $\begin{array}{c}\text { Exhaustive } \\
\text { search }\end{array}$ & 112.8167 & $\checkmark$ & 5.3739 \\
\hline
\end{tabular}

Remark: The Big-M matrices found out using (22) are $m_{C}=-4.15 \times 10^{5}$ and $M_{C}=3.98 \times 10^{5}$. Selecting these matrices achieve global optimal solution in our article. However, in the case where $m_{C}$ (and $M_{C}$ ) are smaller (and larger) values, there may be numerical difficulties in solving SDPs during BNB. In order to avoid this, desired scaling of the system model's input matrix can be performed with the respective scaling of $W_{U}$ in (21) along with descaling of the controller obtained in synthesis.

Figure 3. shows the singular value plots of open loop responses from the disturbances $d_{1} \ldots d_{4}$ to the acceleration outputs $y_{1} \ldots y_{25}$ and compares them with the closed loop responses when all the actuators are used and the closed loop responses when a controller designed from the optimal solution obtained from MBSDP. It can be deduced that the closed loop performs better than the open loop system. Also, it can be easily observed that, both the closed loop responses are similar considering that both solutions add a lot of damping at the resonances. This similarity also shows that, although, the number of actuators are reduced, the performance have not degraded.

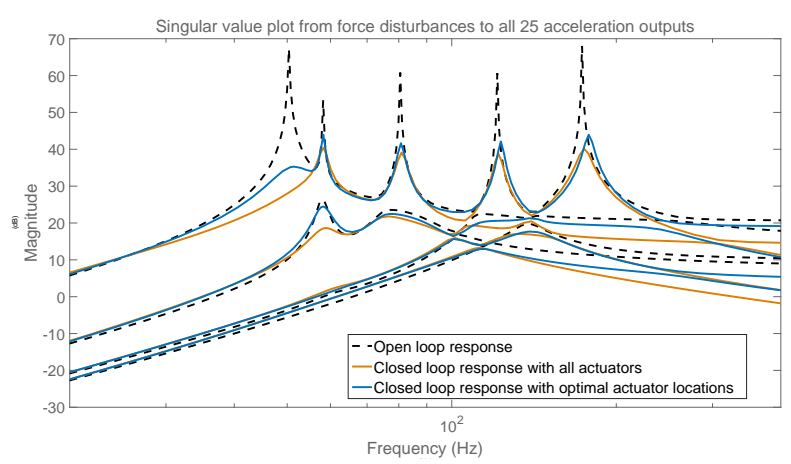

Fig. 3: Singular value plot of the channel from disturbance $d_{1} \ldots d_{4}$ to acceleration outputs $y_{1} \ldots y_{25}$ for comparison between the open loop response and closed loop responses.

Figure 3 gave an insight on the frequency-domain analysis of the closed loop system, while figure 4 shows some timedomain analysis. Here, unit step disturbances are provided to $d_{1} \ldots d_{4}$ and the acceleration outputs $y_{1} \ldots y_{25}$ are recorded. For the sake of comparison, the r.m.s values are plotted for the open loop system, closed loop system with all actuators and closed loop system with the actuators obtained from selection approach. The average reduction of the r.m.s values of the vibration of about $51 \%$ is achieved.

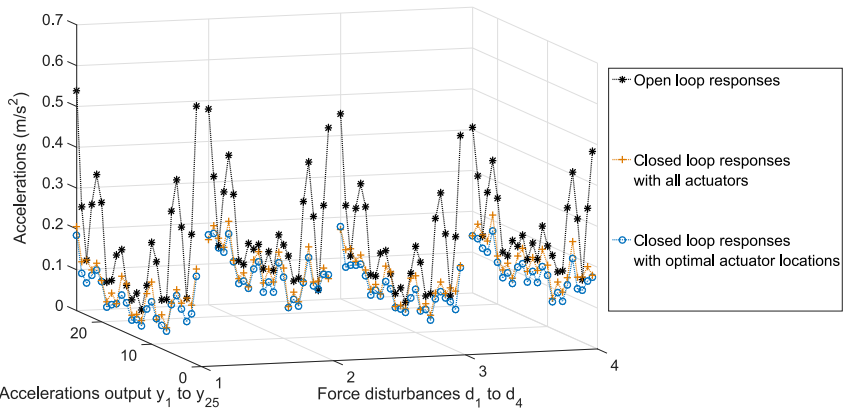

Fig. 4: R.M.S values of accelerations $y_{1} \ldots y_{25}$ from unit step disturbances $d_{1} \ldots d_{4}$ for the open and two closed loop cases.

\section{CONCLUSiON}

This paper discusses two approaches to concurrently design a $\mathcal{H}_{\infty}$ controllers and select optimal sensors and actuators. The first approach formulates the combined problem as a multi-objective problem with iterative reweighted $\ell_{1}$ norm algorithm, which is an heuristic algorithm. The second approach reformulates the problem as a MBSDP problem and uses BNB algorithm to attain the optimal solution.

The discussed approaches are applied to the active vibration control of a smart composite plate consisting of 25 sensors and actuators. The computation time for solving the concurrent design problem using both the approaches is compared with an exhaustive search. Results indicate 
that the heuristics approach is fastest among the compared approaches. Although, MBSDP with BNB is slower than the heuristic approach, it yields global optimal solution: global with respect to closed loop performance and actuator selection (see table. II). Apart from that, the closed loop analyses shows that the global solution reduces the vibrations in the plate similar to when all the actuators are used.

\section{ACKNOWLEDGMENT}

This work is supported by Flanders Make project: SBO ROCSIS: Robust and Optimal Control for Systems of Interacting Subsystems. This work also benefits from KU Leuven Research project C14/15/067: B-spline based certificates of positivity with applications in engineering and KU LeuvenBOF PFV/10/002 Centre of Excellence: Optimization in Engineering (OPTEC).

\section{REFERENCES}

[1] M. Van De Wal and B. De Jager, "A review of methods for input/output selection," Automatica, vol. 37, no. 4, pp. 487-510, 2001.

[2] W. K. Gawronski, Dynamics and control of structures: A modal approach. Springer Science \& Business Media, 2004.

[3] K. Hiramoto, H. Doki, and G. Obinata, "Optimal sensor/actuator placement for active vibration control using explicit solution of algebraic riccati equation," Journal of Sound and Vibration, vol. 229, no. 5, pp. 1057-1075, 2000.

[4] M. Haemers, S. Derammelaere, and K. Stockman, "Co-design of controller and setup configuration using genetic algorithm," in 22nd IEEE International Conference on Emerging Technologies and Factory Automation, 2017.

[5] M. C. De Oliveira and J. Geromel, "Linear output feedback controller design with joint selection of sensors and actuators," IEEE Transactions on Automatic Control, vol. 45, no. 12, pp. 2412-2419, 2000.

[6] K. Hiramoto, J. Mohammadpour, and K. M. Grigoriadis, "Integrated design of system parameters, control and sensor/actuator placement for symmetric mechanical systems," in Decision and Control, 2009 held jointly with the 2009 28th Chinese Control Conference. CDC/CCC 2009. Proceedings of the 48th IEEE Conference on. IEEE, 2009, pp. $2855-2860$.

[7] J. A. Tropp, "Algorithms for simultaneous sparse approximation. part ii: Convex relaxation," Signal Processing, vol. 86, no. 3, pp. 589-602, 2006.

[8] S. Boyd and L. Vandenberghe, Convex optimization. Cambridge university press, 2004.

[9] N. K. Dhingra, M. R. Jovanović, and Z.-Q. Luo, "An ADMM algorithm for optimal sensor and actuator selection," in Decision and Control (CDC), 2014 IEEE 53rd Annual Conference on. IEEE, 2014, pp. 4039-4044.

[10] A. Argha, S. W. Su, A. Savkin, and B. G. Celler, "Mixed $H_{2} / H_{\infty}-$ based actuator selection for uncertain polytopic systems with regional pole placement," International Journal of Control, pp. 1-17, 2017.

[11] T. Singh, J. Swevers, and G. Pipeleers, "Concurrent $\mathrm{H}_{2} / \mathrm{H}_{\infty}$ feedback control design with optimal sensor and actuator selection," in 2018 IEEE 15th International Workshop on Advanced Motion Control (AMC), March 2018, pp. 223-228.

[12] A. Argha, S. W. Su, A. Savkin, and B. Celler, "A framework for optimal actuator/sensor selection in a control system," International Journal of Control, vol. 92, no. 2, pp. 242-260, 2019. [Online]. Available: https://doi.org/10.1080/00207179.2017.1350755

[13] D. G. Luenberger, Y. Ye et al., Linear and nonlinear programming. Springer, 1984, vol. 2.

[14] T. Singh, M. De Mauri, W. Decré, J. Swevers, and G. Pipeleers, "Combined $\mathcal{H}_{\infty} / \mathcal{H}_{2}$ controller design and optimal selection of sensors and actuators," in Joint $9^{\text {th }}$ IFAC Symposium on Robust Control Design and $2^{\text {nd }}$ IFAC Workshop on Linear Parameter Varying Systems, 2018.

[15] S. Nugroho, A. F. Taha, T. Summers, and N. Gatsis, "Simultaneous sensor and actuator selection/placement through output feedback control," in 2018 Annual American Control Conference (ACC), June 2018, pp. 4159-4164.
[16] T. Singh, Z. Dong, M. De Mauri, W. Decré, J. Swevers, and G. Pipeleers, "Combined $\mathcal{H}_{\infty}$ linear parameter varying control design and optimal sensor/actuator selection," in 2019 18th European Control Conference (ECC), June 2019, pp. 3310-3315.

[17] C. Scherer, P. Gahinet, and M. Chilali, "Multiobjective outputfeedback control via LMI optimization," IEEE Transactions on automatic control, vol. 42, no. 7, pp. 896-911, 1997.

[18] E. J. Candès, M. B. Wakin, and S. P. Botd, "Enhancing sparsity by reweighted $\ell 1$ minimization," vol. 14, no. 5-6, pp. 877-905, oct 2008.

[19] Z. Dong, C. Faria, B. Pluymers, M. Hromčík, M. Šebek, and W. Desmet, "Structure-preserving low-order modeling approach of laminated composite plates integrated with macro-fiber composite transducers for dynamic applications," Composite Structures, 2019.

[20] Z. Bai and Y. Su, "Dimension reduction of large-scale second-order dynamical systems via a second-order arnoldi method," Journal of Scientific Computing, vol. 26, pp. 1692-1709, 2005.

[21] M. Verbandt, L. Jacobs, D. Turk, T. Singh, J. Swevers, and G. Pipeleers, "Linear Control Toolbox-supporting B-splines in LPV control," Mechatronics, vol. 52, pp. 78-89, 2018.

[22] J. Lofberg, "YALMIP: A toolbox for modeling and optimization in MATLAB," in Computer Aided Control Systems Design, 2004 IEEE International Symposium on. IEEE, 2004, pp. 284-289.

[23] A. Mosek, "The mosek optimization toolbox for matlab manual," 2015. 